\title{
Intraneural Stimulation Using 2D Wire-Microelectrode Arrays: I. Experimental Results
}

\author{
Jos P.A. Smit, Student Member, IEEE and Wim L.C. Rutten, Member, IEEE \\ Institute for Biomedical Technology, Faculty of Electrical Engineering, University of Twente \\ P.O. Box 217, 7500 AE Enschede, The Netherlands
}

\begin{abstract}
A two-dimensional 24-channel wire-microelectrode array was inserted into the peroneal nerve of the rat during acute experiments. The electrodes in the array are on a regular grid of 6 by 4 electrodes; inter-electrode spacing is $120 \mu \mathrm{m}$. For each of the electrodes in the array the corresponding twitchforce recruitment curve was recorded from the extensor digitorum longus muscle (EDL). A complete set of 24 recruitment curves is presented. The shape of the recruitment curves varies among the electrodes in the array. This supports previous findings which suggest a different motor unit recruitment order for stimulating electrodes at different intraneural positions.
\end{abstract}

\section{INTRODUCTION}

The use of intraneural electrodes is a promising means of increasing selectivity of electrical stimulation. Experiments with (single) intraneural wire electrodes showed that the recruitment behavior in the low-force range depends on the position of the stimulating electrode with respect to the motor fibers $[1,2]$. This was confirmed by a model study [1,2]. To control different (groups of) motor fibers independently, multiple intraneural electrodes will be needed. This implies using electrode arrays.

This paper presents experimental data from intraneural stimulation using a two-dimensional wire-microelectrode array. In the accompanying part II of this paper these data will be compared to experimental data from single-wire electrode experiments.

\section{METHODS}

Acute experiments were conducted on Wistar rats. A twodimensional wire-microelectrode array was inserted into the intact peroneal nerve through an incision. The electrode array consisted of 6 rows of $4 \mathrm{NiCr}$-wire electrodes each. Diameter of the wire electrodes was $25 \mu \mathrm{m}$; interelectrode spacing was $120 \mu \mathrm{m}$. Rectangular depolarizing current pulses of $100 \mu \mathrm{s}$ duration were generated in order to stimulate $\alpha$-motoneurons of the EDL. The elicited twitch forces were measured under isometric conditions.

For each of the electrodes in the array a recruitment curve was recorded by applying stimuli with increasing amplitudes at $1 \mathrm{~s}$ intervals; stimulus current step size was $0.1 \mu \mathrm{A}$. The EDL was allowed 2 minutes of rest between recruitment curves for consecutive electrodes. The peroneal nerve was kept moist by applying Ringer's solution at regular intervals.

\section{RESULTS}

Fig. 1 (next page) shows 24 recruitment curves recorded from one animal for a fixed position of the electrode array. Only twitch-force maxima exceeding $4.9 \mathrm{mN}(0.5 \mathrm{~g})$ are shown, since this value represents the lowest measurable force with the force transducer employed. The curves were recorded sequentially, from electrode 1 to electrode 24 . Note that the curves are plotted on a double-logarithmic scale.

Recruitment curves from different electrodes have different shapes; this is especially apparent in the low-force range. As an example, the curves for electrodes 16 and 17 show a gradual increase of force with stimulation current, while no (or only small) force plateaus are visible. Electrode 18 (which is separated $120 \mu \mathrm{m}$ from electrode 17), however, shows a large force plateau at approximately $15 \mathrm{mN}$.

In general, the data in Fig. 1 show different recruitment patterns for different electrodes. This is reflected by differences in force step size and existence (or absence) of force plateaus among curves for different electrodes.

\section{Discussion}

The observed differences in recruitment curve shapes support previous findings [1,2] which suggest a different motor unit recruitment order when stimulating with electrodes at different intraneural positions.

To find the order in which motor units are recruited for each electrode, motor unit forces have to be extracted first from the recruitment curves. This involves identifying force steps that are common among several recruitment curves, and then extracting additional force steps in a combinatorial analysis.

\section{REFERENCES}

[1] J.P.A. Smit, W.L.C. Rutten, T.A. Frieswijk, and R. Busschers, "The influence of nerve fiber distribution on the recruitment behavior resulting from intraneural stimulation," in Proc. 15th Ann. Int. Conf. IEEE Eng. Med. Biol. Soc., pp. 1198-1199, 1993.

[2] W.L.C. Rutten, T.A. Frieswijk, J.P.A Smit, T.H. Rozijn, and J.H. Meier, "3D Neuro-electronic interface devices for neuromuscular control: Design studies and realisation steps," Biosensors \& Bioelectronics, vol. 10, pp. 141-153, 1995. 

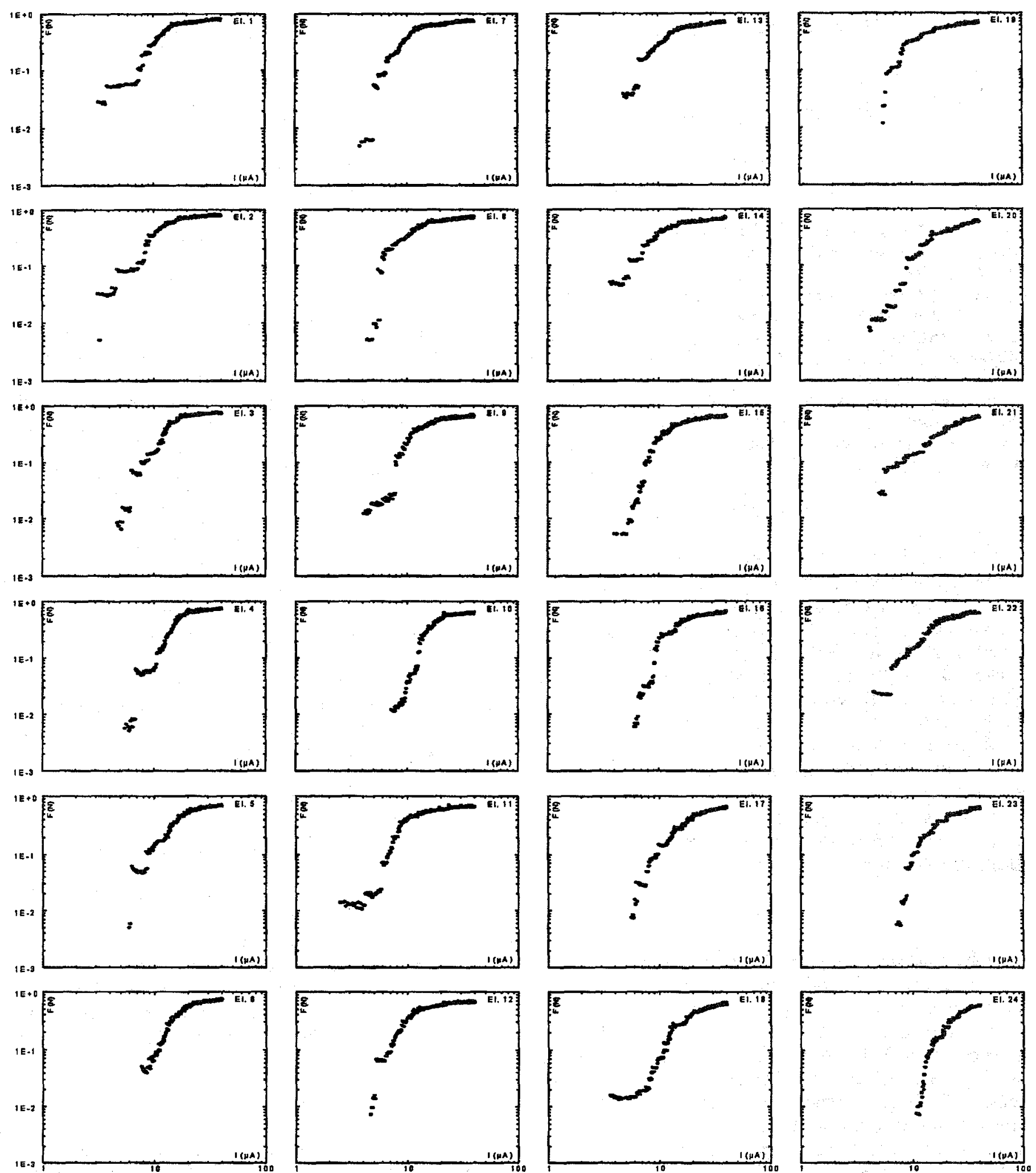

Fig. 1. Recruitment curves obtained from one animal for a fixed position of the 24-channel wire-microelectrode array. Each curve is the result of stimulation with a single electrode. The electrodes in the array are on a regular grid of 6 by 4 electrodes; inter-electrode spacing is $120 \mu \mathrm{m}$. Electrode numbers are indicated in the upper right corner for each curve. Note that the curves are plotted on a double-logarithmic scale (indicated scales apply to all curves). Horizontal axis: stimulation current I; vertical axis: twitch-force maximum F. Top to bottom: recruitment curves for electrodes positioned along the longitudinal direction of the peroneal nerve (top: proximal; bottom: distal). Left to right: curves for electrodes positioned along the radial direction of the peroneal nerve (left: medial; right: lateral). 